\title{
Development and reproducibility of a computed tomography-based measurement of renal sinus fat
}

\author{
Meredith C Foster ${ }^{1,2,3}$, Shih-Jen Hwang ${ }^{1,3}$, Stacy A Porter ${ }^{4}$, Joseph M Massaro ${ }^{5}$, Udo Hoffmann ${ }^{6}$ and \\ Caroline S Fox ${ }^{1,3,7^{*}}$
}

\begin{abstract}
Background: Renal sinus fat may mediate obesity-related vascular disease, although this fat depot has not been assessed in a community-based sample. We sought to develop a protocol to quantify renal sinus fat accumulation using multi-detector computed tomography (MDCT).

Methods: Protocol development was performed in participants in the Framingham Offspring cohort who underwent MDCT. Volumetric renal sinus fat was measured separately within the right and left kidneys, and renal sinus fat area within a single MDCT scan slice was measured in the right kidney. Due to the high correlation of volumetric and single-slice renal sinus fat in the right kidney (Pearson correlation $[r]=0.85, p<0.0001$ ), we optimized a single-slice protocol to capture renal sinus fat in the right kidney alone. Pearson correlation coefficients were used to compare to assess the correlation of volumetric and single-slice renal sinus fat in the right kidney with other measures of adiposity. Inter- and intra-reader reproducibility was assessed using intra-class correlation coefficients.
\end{abstract}

Results: Single-slice measurements were obtained in 92 participants (mean age 60 years, 49\% women, median renal sinus fat $0.43 \mathrm{~cm}^{2}$ ). Intra- and inter-reader intra-class correlation coefficients were 0.93 and 0.86 , respectively. Single-slice renal sinus fat was correlated with body mass index $(r=0.35, \mathrm{p}=0.0006)$, waist circumference $(r=$ $0.31, p=0.003)$, and abdominal visceral fat $(r=0.48, p<0.0001)$. Similar correlations were observed for volumetric renal sinus fat in the right kidney.

Conclusions: Measuring renal sinus fat is feasible and reproducible using MDCT scans in a community-based sample.

\section{Background}

Chronic kidney disease (CKD) is a major global health concern $[1,2]$. CKD affects over $13 \%$ of adults in the United States [3] and recent estimates in European adults indicate that stage 3 to 5 CKD affects about 4-7\% of men and $6-10 \%$ of women [4]. CKD is associated with cardiovascular disease [5,6] and increased mortality $[7,8]$ in addition to diabetes, hypertension, smoking, and obesity [9-16].

Obesity has reached epidemic proportions, with an estimated 1.3 billion adults worldwide either overweight or obese [17]. In the United States, the prevalence of obesity has continued to increase over the past 50 years [18],

\footnotetext{
* Correspondence: foxca@nhlbi.nih.gov

'National Heart, Lung, and Blood Institute's Framingham Heart Study, 73 Mt. Wayte Avenue, Suite 2, Framingham, Massachusetts, 01702 USA

Full list of author information is available at the end of the article
}

and the most recent reports indicate that one-third of American adults are obese, with an additional one-third overweight [19]. In 2002, the prevalence of obesity was greater than $20 \%$ in at least half of the countries in the European Union [20]. While obesity as defined based on body mass index (BMI) is associated with the development of CKD [12-16], reports indicate that measures of abdominal adiposity are also associated with CKD [21-24]. The observed associations of abdominal adiposity with CKD suggest that regional fat accumulation and ectopic fat, defined as the accumulation of fat within and around non-adipose tissues and organs [25], may mediate the relation of obesity to renal function. Within the kidney, ectopic fat can accumulate within the renal sinus, a renal cavity that also contains the renal artery, renal vein, lymphatic vessels, and nerves and has been observed in humans [26-28] and in an animal model of diet-induced
C Biomed Central 
obesity [29]. It is hypothesized to impair renal function through compression of renal structures, the release of locally acting molecules, or lipotoxicity in renal tissue.

Because the effects of such organ-specific fat accumulation are not captured using traditional anthropometric measurements of adiposity, radiographic techniques are necessary to investigate the potential role of kidney-specific fat accumulation in renal function. In the Framingham Heart Study, we have collected abdominal multi-detector computed tomography (MDCT) scans in a large sample of participants from the Offspring and Third Generation cohorts and have visualized the renal sinus. However, the feasibility and reproducibility of a MDCT-based renal sinus fat measurement has not been well-studied in a community-based sample. Thus, we sought to develop a method to measure renal sinus fat accumulation using MDCT in a sample from the Framingham Heart Study and to assess its reproducibility.

\section{Methods}

\section{Study sample}

The original Framingham Heart Study cohort was established in 1948 and consisted of 5209 adults between 28 and 62 years old from Framingham, Massachusetts. The Offspring cohort was established in 1971 and consisted of 5124 children and spouses of children of the original cohort. The Third Generation cohort was established in 2002 and consisted of 4095 participants with at least one parent in the Offspring cohort. The Framingham MDCT cohort consists of 3539 participants from the Offspring $(n=1422)$ and Third Generation $(n=2117)$ cohorts who had a MDCT scan between June 2002 and March 2005, as previously described [30]. A sample of 100 Offspring cohort participants from the MDCT cohort was used for the present analysis and was selected for equal representation of sex and 10-year age groups (35-44, 45-54, 55-64, 65-74, and 75-84 years old). Participants were randomly selected within strata defined by sex and age group. Participants provided written informed consent and this study was approved by the Boston University Medical Center and Massachusetts General Hospital institutional review boards and was conducted in accordance with the Helsinki Declaration.

\section{MDCT Scan Acquisition}

Computed tomography images were captured using an 8slice MDCT scanner (LightSpeed Ultra, General Electric; Milwaukee, WI, USA). The MDCT scan covered $125 \mathrm{~mm}$ in the abdomen with $255.0-\mathrm{mm}$ slices above the S1 level $(120 \mathrm{kVp}, 400 \mathrm{~mA}$, gantry rotation time $500 \mathrm{~ms}$, table feed 3:1). Management and interpretation of MDCT scans was performed on a dedicated terminal using the Aquarius 3D Workstation (TeraRecon, Inc, San Mateo, CA, USA).

\section{Protocol Development}

Renal sinus fat accumulation was measured using the Aquarius 3D Workstation body fat analysis template. Pixel density in Hounsfield units (HU) was used to identify adipose tissue based on a window width of -195 to $-45 \mathrm{HU}$, centered on $-120 \mathrm{HU}$.

The abdominal MDCT scans were originally collected to assess abdominal aortic calcification. Because of this, the entire kidneys were not visualized in our participants. Several characteristics suggested that enough of the right, but not the left kidney, was visible in our scans to measure renal sinus fat. We captured more of the right kidney than the left kidney on average in our study sample (14.6 versus 13.9 slices, respectively, $\mathrm{p}=0.02$ ). Additionally, we observed that volumetric renal sinus fat in the left kidney was correlated with the number of slices captured in the left kidney (Pearson correlation coefficient $[r]=0.29, \mathrm{p}=0.005)$ whereas volumetric renal sinus fat in the right kidney was not $(r=0.11, \mathrm{p}=0.27)$. This correlation in the left kidney was a concern, because it indicated that the measurement in the left kidney may be a function of the amount of kidney captured in the MDCT scans and may not reflect a true estimate of overall renal sinus fat in the left kidney. Based on these observations, we selected only the right kidney for further protocol development.

The next step in protocol development was to determine if a single slice that captures the renal sinus in the right kidney could represent volumetric renal sinus fat accumulation in the right kidney. In order to answer this question, we measured renal sinus fat area in a single slice from the right kidney and compared it to volumetric renal sinus fat measured using all visualized slices of the right kidney. The single-slice area measurement was correlated with the volumetric measurement in the right kidney $(r=0.85, \mathrm{p}<0.0001)$. Thus, we further optimized this single-slice protocol and developed a selection rule to identify the final renal sinus fat measurement slice.

Our final protocol consisted of a single-slice area measurement of renal sinus fat in the right kidney. To determine the slice with the maximum amount of fat within the renal sinus, the reader selected a range of several scan slices that appeared to have equivalent amounts of fat based on visual inspection. The single measurement slice was selected from this range using the following selection rule: if the range included an odd number of slices, then the middle slice of the range was traced; if the range included an even number of slices, then the anatomically more cranial of the two middle slices was traced. An example of a selected slice is presented in Figure 1a. The outer edge of the kidney in the selected slice was manually traced to separate it from the rest of the abdominal cavity (Figure 1b). The reader traced within the border of the kidney such that surrounding abdominal adipose tissue 
would be excluded from this measurement. The reader used a straight-line trace across the opening of the renal sinus from the dimples in the two adjacent lobes based on visual inspection (Figure 1b). Once traced, the Aquarius 3D Workstation software was used to measure the fat area within one slice of the right kidney (Figure 1c). Participants were excluded if (1) the renal sinus was not visualized or (2) if cysts or other structural abnormalities were present in the right kidney $(n=8)$.

\section{Covariate assessment}

BMI $\left(\mathrm{kg} / \mathrm{m}^{2}\right)$ and waist circumference $(\mathrm{cm})$ were determined based on anthropometric measurements taken by the trained clinic staff during the seventh Offspring examination cycle. During the clinic visit, weight was measured to the nearest pound, and height and waist circumference at the level of the umbilicus were measured to the nearest quarter-inch. Volumetric abdominal subcutaneous $\left(\mathrm{SAT}, \mathrm{cm}^{3}\right.$ ) and visceral adipose tissue $\left(\mathrm{VAT}, \mathrm{cm}^{3}\right)$ were assessed using the same set of abdominal MDCT scans, as previously described [31].

\section{Statistical methods}

The final single-slice protocol was performed separately by two readers (MCF, SAP) for inter-observer variation and then repeated by the first reader (MCF) for intraobserver variation. Inter- and intra-reader reproducibility was assessed using intra-class correlation coefficients. Bland-Altman plots were utilized to assess potential systematic biases within the intra-reader and inter-reader repeated measurements. Correlations of single-slice renal sinus fat and volumetric renal sinus fat in the right kidney with BMI, waist circumference, SAT, and VAT were assessed using age- and sex-adjusted partial Pearson correlation coefficients. Statistical analyses were performed using SAS Version 9.2 (SAS Institute Inc; Cary, NC, USA).

\section{Results}

\section{Sample characteristics}

Among the 100 individuals in the reproducibility sample, 92 participants had abdominal MDCT scans that could be used for the single-slice measurement protocol. Characteristics of the reproducibility sample with renal sinus fat measurements are presented in Table 1 . The mean age was 60 years and $52 \%(n=48)$ were women. The mean BMI, waist circumference, and abdominal VAT volume were $27.7 \mathrm{~kg} / \mathrm{m}^{2}, 98.7 \mathrm{~cm}$, and $2007 \mathrm{~cm}^{3}$, respectively.

\section{Distribution of renal sinus fat}

Among participants with a volumetric renal sinus fat measurement in the right kidney $(\mathrm{n}=91)$, the mean


Figure 1 Abdominal computed tomography scans demonstrating the renal sinus fat measurement technique. $A$ : Example of single abdominal computed tomography slice selected for renal sinus fat measurement. B: One measurement was taken in the right kidney. The reader manually traced within the border of the kidney. C: Visualization of renal sinus fat in slice measured in B using the Aquarius Workstation body fat analysis template (TeraRecon, Inc, San Mateo, CA, USA). The renal sinus is traced in this figure, highlighting the visualization of fat tissue observed in this region of interest. 
Table 1 Demographic characteristics of the reproducibility sample at the $7^{\text {th }}$ Offspring Examination

\begin{tabular}{ccccccccc}
\hline Variable & $\mathbf{N}$ & Mean & Standard Deviation & Minimum & $\mathbf{2 5}^{\text {th }}$ percentile & Median & $\mathbf{7 5}^{\text {th }}$ percentile & Maximum \\
\hline Age (years) & 92 & 60 & 13 & 37 & 48 & 60 & 72 & 83 \\
\hline Weight $(\mathbf{k g})$ & 92 & 78.8 & 16.0 & 48.5 & 67.1 & 79.4 & 90.5 & 137.0 \\
\hline Height $(\mathbf{c m})$ & 92 & 168.4 & 9.0 & 150.5 & 160.0 & 168.0 & 175.6 & 190.5 \\
\hline Body mass index $\left(\mathbf{k g} / \mathbf{m}^{\mathbf{2}}\right)$ & 92 & 27.7 & 4.6 & 18.1 & 24.6 & 27.3 & 30.0 & 41.5 \\
\hline Waist circumference $(\mathbf{c m})$ & 92 & 98.7 & 12.7 & 71.8 & 89.9 & 99.1 & 106.4 & 132.1 \\
\hline Subcutaneous Adipose Tissue $\left.\mathbf{( c m}^{\mathbf{3}}\right)$ & 92 & 2930 & 1291 & 501 & 1971 & 2743 & 3410 & 6695 \\
\hline Visceral Adipose Tissue $\left(\mathbf{c m}^{\mathbf{3}}\right)$ & 92 & 2007 & 1025 & 289 & 1249 & 1882 & 2758 \\
\hline Renal fat measures & & & & & & & & \\
\hline Right kidney, volumetric $\left(\mathbf{c m}^{\mathbf{3}}\right)$ & 91 & 5.62 & 5.40 & 0.0095 & 1.83 & 3.99 & 7.49 \\
\hline Right kidney, one-slice $\left(\mathbf{c m}^{\mathbf{2}}\right)$ & 92 & 0.69 & 0.72 & $<0.0048$ & 0.17 & 0.43 & 1.07 \\
\hline
\end{tabular}

total fat volume was $5.62 \mathrm{~cm}^{3}$, ranging from $0.01 \mathrm{~cm}^{3}$ to $25.61 \mathrm{~cm}^{3}$. No participants had an undetectable volumetric renal sinus fat measurement in the right kidney; the volumetric renal sinus fat measurement was missing for one participant. Among participants with an interpretable MDCT scan for the single-slice renal sinus fat measurement $(n=92)$, the mean renal sinus fat area was $0.69 \mathrm{~cm}^{2}$, ranging from undetectable $(\mathrm{n}=3)$ to $2.95 \mathrm{~cm}^{2}$.

\section{Intra- and inter-reader reproducibility}

Intra- and inter-reader renal fat measurements are plotted in Figures 2a and 2b, respectively. The intrareader intra-class correlation coefficient was 0.93 and the inter-reader intra-class correlation coefficient was 0.86 for our single-slice measurement protocol.

The Bland-Altman plot for the intra-reader repeated measurements is presented in Figure 3A. Overall, the mean difference between the repeated measurements was $0.018 \mathrm{~cm}^{2}$ with upper and lower confidence limits of $0.435 \mathrm{~cm}^{2}$ and $-0.470 \mathrm{~cm}^{2}$, respectively, suggesting minimal systematic bias between the intra-reader measurements. The Bland-Altman plot for the inter-reader repeated measurements is presented in Figure 3B. Here, the mean difference between the repeated measures was $0.157 \mathrm{~cm}^{2}$, with upper and lower confidence limits of $0.299 \mathrm{~cm}^{2}$ and $-0.613 \mathrm{~cm}^{2}$, indicating that the first reader's measurements are on average slightly higher than the second reader's measurements. While this suggests a systematic bias in the inter-reader measurements, our main objective was to develop a single-slice protocol to use as a marker of overall renal sinus fat accumulation. This bias would likely not impact the ranking of individuals by renal sinus fat accumulation within each set of reader measurements.

Correlation of renal sinus fat with adiposity measures Age- and sex-adjusted partial Pearson correlation coefficients for single-slice renal sinus fat and volumetric

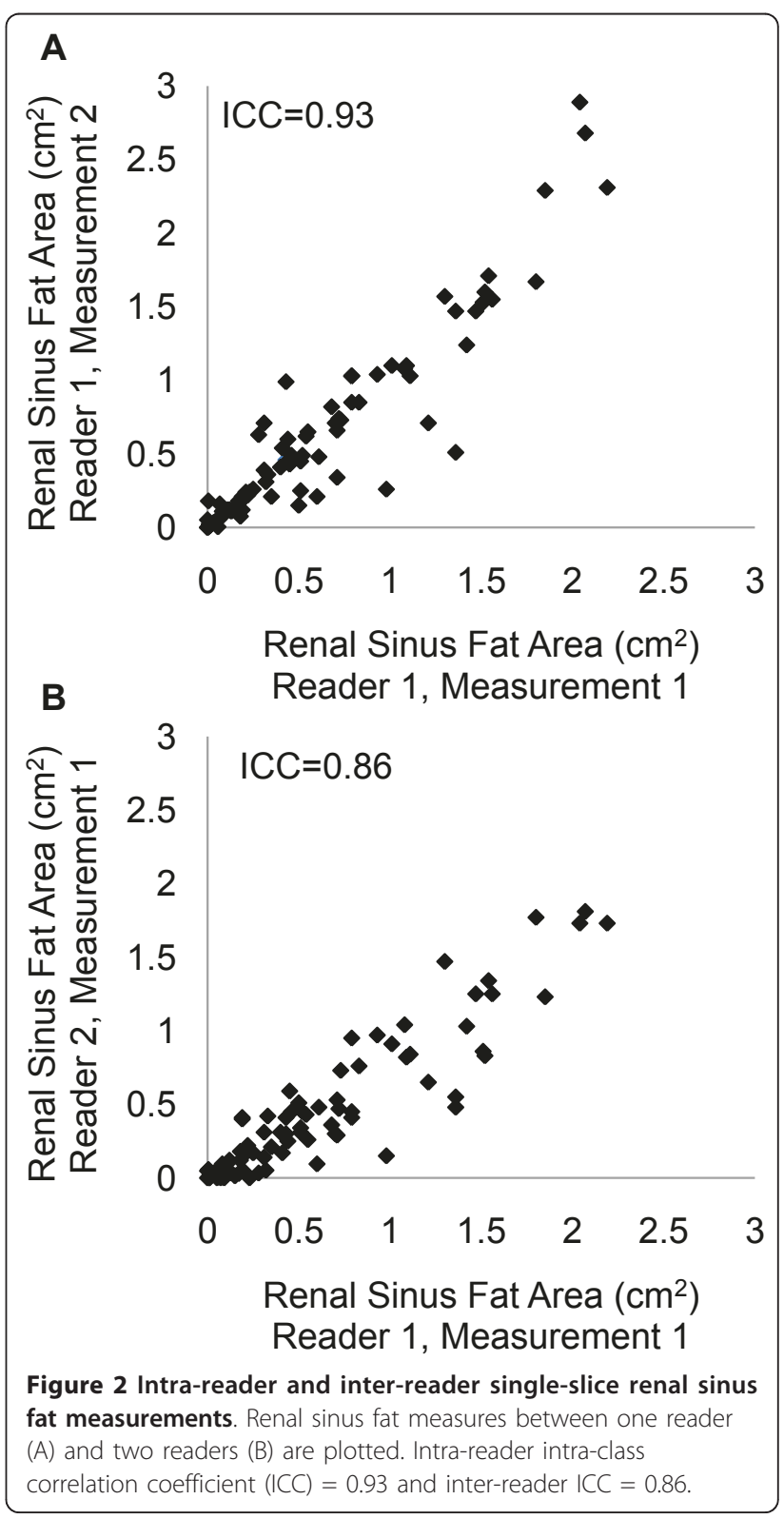



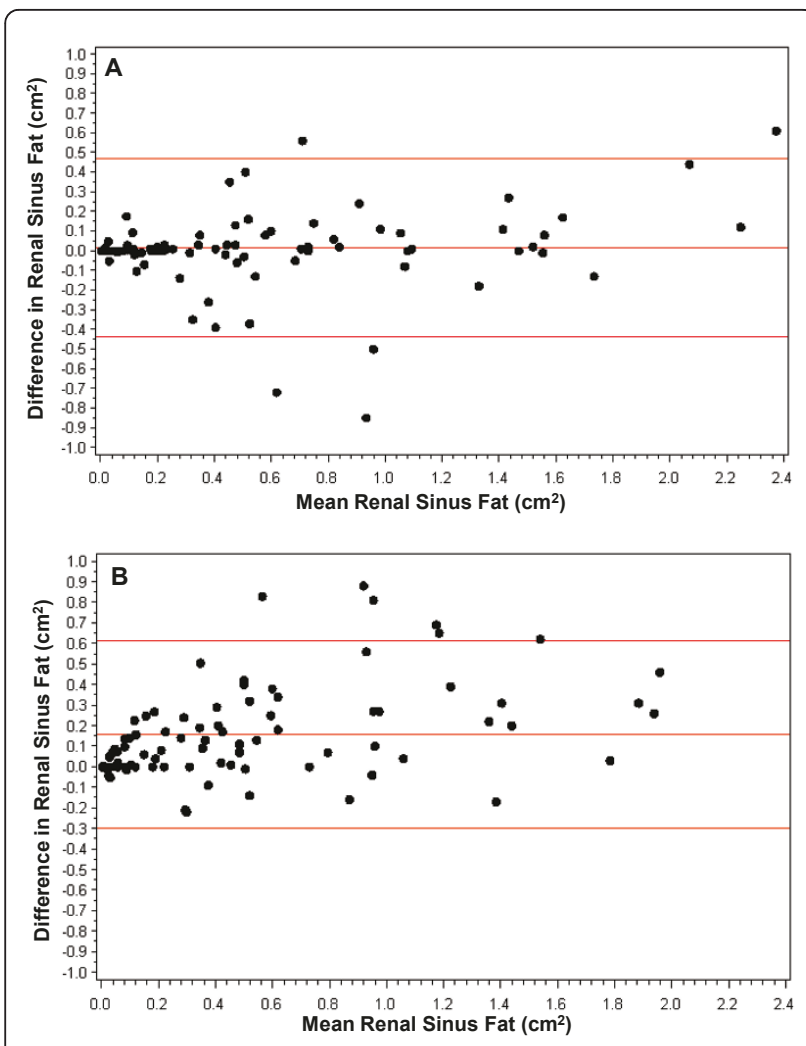

Figure 3 Bland-Altman plots of $(A)$ the intra-reader renal sinus fat measurements and (B) the inter-reader renal sinus fat area measurements. The average of the repeated measurements is presented on the $\mathrm{X}$-axis and the difference between the two measurements is presented on the $Y$-axis. The middle red line represents the mean difference between the repeated measures and the upper and lower red lines represent upper and lower confidence limits for the mean difference, respectively.

renal sinus fat in the right kidney with measures of adiposity are presented in Table 2 . Single-slice renal sinus fat was correlated with BMI $(r=0.35)$, waist circumference $(r=0.31)$, and VAT $(r=0.48$, all $p \leq$ 0.003). Similar correlations were observed for volumetric renal sinus fat.

\section{Discussion}

Using a sample from the Framingham MDCT study cohort, we have shown that measuring renal sinus fat using computed tomography is feasible and reproducible in a community-based sample. During protocol development, we observed that a single-slice renal sinus fat measurement is highly correlated with volumetric renal sinus fat accumulation in the right kidney. We also observed that the single-slice and volumetric renal sinus fat measurements are similarly correlated with other measures of adiposity, including BMI, waist circumference, and abdominal VAT, suggesting that only one slice needs to be manually traced by the reader to assess renal sinus fat.

Computed tomography, among other imaging techniques, has been used to assess the development of renal lesions in humans, including renal sinus fat accumulation $[32,33]$. Previous small imaging studies using magnetic resonance imaging have demonstrated fat accumulation in the renal sinus of children with no known renal disease ( $n=15$ of 58 participants) [26] and adults [27]. Magnetic resonance imaging has also been used to assess renal sinus fat in 205 participants in the Pulmonary Edema and Stiffness of the Vascular System study, which consists of middle-aged and elderly participants with cardiovascular risk factors [28]. In the animal literature, renal sinus fat accumulation has been described in a rabbit model of diet-induced obesity [25,29]. In obese rabbits fed a high fat ad lib diet for 8-12 weeks, renal sinus mass was $61 \%$ greater than observed in lean control rabbits, with the majority of this increase due to a 2.6 -fold increase in renal sinus fat accumulation [29]. It is proposed that such increases in renal sinus fat can compress renal structures, including the renal medulla, renal vein and lymph vessels, leading to changes in renal interstitial pressure [25]. Renal sinus fat may also lead to renal tissue damage through paracrine effects of locally released adipocytokines or lipotoxicity.

Lipid accumulation within renal tissues has also been observed in humans [34,35] and animal models [36-40]. The results from animal models support mechanisms of obesity-induced hypertension and renal dysfunction through oxidative stress, inflammation, and fibrosis within glomeruli and proximal tubules [41,42]. While renal lipid accumulation is important when considering the impact of obesity on renal function, this accumulation within renal cells is not captured in our measurement of renal sinus fat. Other techniques, including magnetic resonance imaging [43], are required to address renal intra-cellular lipid accumulation and its potential role in obesity-related renal dysfunction in humans.

The present study adds to the literature with the development of a protocol to quantify the accumulation of renal sinus fat in a community-based sample based on imaging techniques. Our study demonstrates that computed tomography can be used to develop a reproducible measurement protocol for renal sinus fat accumulation. Given the current obesity epidemic, ectopic fat depots may play an important role in organ dysfunction. This technique will allow for the characterization of the distribution of renal sinus fat and to assess its correlation with other clinical characteristics, which may lead to a better understanding of the role of adiposity in renal dysfunction and obesity-related vascular disease.

A major strength of our study is the use of a wellcharacterized, community-based sample that was not 
Table 2 Age- and sex-adjusted partial Pearson correlation coefficients of renal sinus fat with adiposity measures in the reproducibility sample

\begin{tabular}{lcc}
\hline & $\begin{array}{c}\text { Volumetric Renal Sinus Fat } \\
\mathbf{( n = 9 1 )}\end{array}$ & $\begin{array}{c}\text { Single-Slice Renal Sinus Fat } \\
(\mathbf{n}=\mathbf{9 2})\end{array}$ \\
\hline Body Mass Index & 0.35 & 0.35 \\
& $p=0.0008$ & $p=0.0006$ \\
\hline Waist Circumference & 0.32 & 0.31 \\
& $p=0.002$ & $p=0.003$ \\
\hline Subcutaneous Adipose Tissue & 0.17 & 0.17 \\
\hline Visceral Adipose Tissue & $p=0.12$ & $p=0.11$ \\
& 0.49 & 0.48 \\
\end{tabular}

selected for obesity-related outcomes. There are limitations of our study that warrant mention. No in vivo gold standard measurement for renal sinus fat exists in human studies, which limits our ability to assess measurement validity. Our reproducibility sample is comprised of white participants from the Framingham Heart Study, which may limit the generalizability of our findings to other cohorts or ethnicities.

\section{Conclusions}

In conclusion, renal sinus fat accumulation can be reproducibly measured using computed tomography in a sample from the Framingham MDCT cohort. In our protocol development, we observed that a single-slice measurement of renal sinus fat accumulation is correlated with volumetric renal sinus fat accumulation in the right kidney.

\section{Acknowledgements}

The Framingham Heart Study is supported by the National Heart, Lung and Blood Institute (N01-HC-25195). The funding body did not play a role in the study design, collection, analysis, and interpretation of data, in the writing of the manuscript, or the decision to submit the manuscript for publication.

\section{Author details \\ ${ }^{1}$ National Heart, Lung, and Blood Institute's Framingham Heart Study, 73 Mt. Wayte Avenue, Suite 2, Framingham, Massachusetts, 01702 USA. 2Department of Epidemiology, Harvard School of Public Health, Boston, Massachusetts, USA. ${ }^{3}$ Center for Population Studies, National Heart, Lung, and Blood Institute, National Institutes of Health, Bethesda, Maryland, USA. ${ }^{4}$ Harvard Medical School, 25 Shattuck Street, Boston, Massachusetts, 02115, USA. ${ }^{5}$ Department of Biostatistics, Boston University School of Public Health, 715 Albany Street, T4E, Boston, Massachusetts, 02118 USA. ${ }^{6}$ Department of Radiology, Massachusetts General Hospital, 55 Fruit Street, Boston, Massachusetts, 02114 USA. 7 Division of Endocrinology and Metabolism, Brigham and Women's Hospital, Harvard Medical School, Boston, Massachusetts, USA.}

\section{Authors' contributions}

MCF contributed to the conception and design of this study, analysis and interpretation of data, and drafting the manuscript. SJH contributed to the analysis and interpretation of data and revising the article critically for important intellectual content. SAP participated in data acquisition and revising the manuscript critically for important intellectual content. JMM and $\mathrm{UH}$ revising the manuscript critically for important intellectual content. CSF contributed to the conception and design of this study, analysis and interpretation of data, and drafting the manuscript and revising the manuscript for important intellectual content. All authors read and approved the final manuscript.

\section{Competing interests}

The authors declare that they have no competing interests.

Received: 14 March 2011 Accepted: 4 October 2011

Published: 4 October 2011

\section{References}

1. Eknoyan G, Lameire N, Barsoum R, Eckardt KU, Levin A, Levin N, et al: The burden of kidney disease: improving global outcomes. Kidney Int 2004, 66:1310-1314.

2. Levey AS, Atkins R, Coresh J, Cohen EP, Collins AJ, Eckardt KU, et al: Chronic kidney disease as a global public health problem: approaches and initiatives - a position statement from Kidney Disease Improving Global Outcomes. Kidney Int 2007, 72:247-259.

3. Coresh J, Selvin E, Stevens LA, Manzi J, Kusek JW, Eggers P, et al: Prevalence of chronic kidney disease in the United States. JAMA 2007, 298:2038-2047.

4. Zoccali C, Kramer A, Jager KJ: Chronic kidney disease and end-stage renal disease: a review produced to contribute to the report 'the status of health in the European Union: towards a healthier Europe. NDT Plus 2010, 3:213-224.

5. Go AS, Chertow GM, Fan D, McCulloch CE, Hsu CY: Chronic kidney disease and the risks of death, cardiovascular events, and hospitalization. $N$ Engl J Med 2004, 351:1296-1305.

6. Anavekar NS, McMurray JJ, Velazquez EJ, Solomon SD, Kober L, Rouleau JL, et al: Relation between renal dysfunction and cardiovascular outcomes after myocardial infarction. N Engl J Med 2004, 351:1285-1295.

7. Weiner DE, Tighiouart H, Amin MG, Stark PC, MacLeod B, Griffith $J$, et al: Chronic kidney disease as a risk factor for cardiovascular disease and allcause mortality: a pooled analysis of community-based studies. J Am SOC Nephrol 2004, 15:1307-1315.

8. Culleton BF, Larson MG, Wilson PW, Evans JC, Parfrey PS, Levy D: Cardiovascular disease and mortality in a community-based cohort with mild renal insufficiency. Kidney Int 1999, 56:2214-2219.

9. Longenecker JC, Coresh J, Powe NR, Levey AS, Fink NE, Martin A, et al: Traditional cardiovascular disease risk factors in dialysis patients compared with the general population: the CHOICE Study. J Am SOC Nephrol 2002, 13:1918-1927.

10. Wheeler DC, Townend JN, Landray MJ: Cardiovascular risk factors in predialysis patients: baseline data from the Chronic Renal Impairment in Birmingham (CRIB) study. Kidney Int Supp/ 2003, S201-S203.

11. Yamagata K, Ishida K, Sairenchi T, Takahashi H, Ohba S, Shiigai T, et al: Risk factors for chronic kidney disease in a community-based population: a 10-year follow-up study. Kidney Int 2007, 71:159-166.

12. Fox CS, Larson MG, Leip EP, Culleton B, Wilson PW, Levy D: Predictors of new-onset kidney disease in a community-based population. JAMA 2004, 291:844-850.

13. Iseki K, Ikemiya Y, Kinjo K, Inoue T, Iseki C, Takishita S: Body mass index and the risk of development of end-stage renal disease in a screened cohort. Kidney Int 2004, 65:1870-1876. 
14. Gelber RP, Kurth T, Kausz AT, Manson JE, Buring JE, Levey AS, et al: Association between body mass index and CKD in apparently healthy men. Am J Kidney Dis 2005, 46:871-880.

15. Hsu CY, McCulloch CE, Iribarren C, Darbinian J, Go AS: Body mass index and risk for end-stage renal disease. Ann Intern Med 2006, 144:21-28.

16. Foster MC, Hwang SJ, Larson MG, Lichtman JH, Parikh NI, Vasan RS, et al: Overweight, Obesity, and the Development of Stage 3 CKD: The Framingham Heart Study. Am J Kidney Dis 2008, 52:39-48.

17. Kelly T, Yang W, Chen CS, Reynolds K, He J: Global burden of obesity in 2005 and projections to 2030. Int J Obes (Lond) 2008, 32:1431-1437.

18. Ogden CL, Yanovski SZ, Carroll MD, Flegal KM: The epidemiology of obesity. Gastroenterology 2007, 132:2087-2102.

19. Ogden CL, Carroll MD, Curtin LR, McDowell MA, Tabak CJ, Flegal KM: Prevalence of overweight and obesity in the United States, 1999-2004. JAMA 2006, 295:1549-1555.

20. Fry J, Finley W: The prevalence and costs of obesity in the EU. Proc Nutr Soc 2005, 64:359-362.

21. Chen J, Muntner P, Hamm LL, Jones DW, Batuman V, Fonseca V, et al: The metabolic syndrome and chronic kidney disease in U.S. adults. Ann Intern Med 2004, 140:167-174.

22. Kurella M, Lo JC, Chertow GM: Metabolic syndrome and the risk for chronic kidney disease among nondiabetic adults. J Am Soc Nephrol 2005, 16:2134-2140.

23. Lee JE, Choi SY, Huh W, Kim YG, Kim DJ, Oh HY: Metabolic syndrome, Creactive protein, and chronic kidney disease in nondiabetic, nonhypertensive adults. Am J Hypertens 2007, 20:1189-1194.

24. Elsayed EF, Sarnak MJ, Tighiouart H, Griffith JL, Kurth T, Salem DN, et al: Waist-to-Hip Ratio, Body Mass Index, and Subsequent Kidney Disease and Death. Am J Kidney Dis 2008.

25. Montani JP, Carroll JF, Dwyer TM, Antic V, Yang Z, Dulloo AG: Ectopic fat storage in heart, blood vessels and kidneys in the pathogenesis of cardiovascular diseases. Int J Obes Relat Metab Disord 2004, 28(Suppl 4): S58-S65.

26. Dietrich $\mathrm{RB}$, Kangarloo $\mathrm{H}$ : Kidneys in infants and children: evaluation with MR. Radiology 1986, 159:215-221.

27. Hricak H, Crooks L, Sheldon $P$, Kaufman L: Nuclear magnetic resonance imaging of the kidney. Radiology 1983, 146:425-432.

28. Chughtai HL, Morgan TM, Rocco M, Stacey B, Brinkley TE, Ding J, et al: Renal sinus fat and poor blood pressure control in middle-aged and elderly individuals at risk for cardiovascular events. Hypertension 2010, 56:901-906.

29. Dwyer TM, Mizelle HL, Cockrell K, Buhner P: Renal sinus lipomatosis and body composition in hypertensive, obese rabbits. Int $J$ Obes Relat Metab Disord 1995, 19:869-874.

30. Fox CS, Massaro JM, Hoffmann U, Pou KM, Maurovich-Horvat P, Liu CY, et al: Abdominal visceral and subcutaneous adipose tissue compartments: association with metabolic risk factors in the Framingham Heart Study. Circulation 2007, 116:39-48.

31. Maurovich-Horvat P, Massaro J, Fox CS, Moselewski F, O'Donnell CJ, Hoffmann U: Comparison of anthropometric, area- and volume-based assessment of abdominal subcutaneous and visceral adipose tissue volumes using multi-detector computed tomography. Int J Obes (Lond) 2007, 31:500-506.

32. Rha SE, Byun JY, Jung SE, Oh SN, Choi YJ, Lee A, et al: The renal sinus: pathologic spectrum and multimodality imaging approach. Radiographics 2004, 24(Suppl 1):S117-S131.

33. Nikolaidis P, Gabriel H, Khong K, Brusco M, Hammond N, Yagmai V, et al: Computed tomography and magnetic resonance imaging features of lesions of the renal medulla and sinus. Curr Probl Diagn Radiol 2008, 37:262-278.

34. Kimmelstiel P, Wilson C: Intercapillary Lesions in the Glomeruli of the Kidney. Am J Pathol 1936, 12:83-98.

35. Lee HS, Lee JS, Koh HI, Ko KW: Intraglomerular lipid deposition in routine biopsies. Clin Nephrol 1991, 36:67-75.

36. Jiang T, Wang Z, Proctor G, Moskowitz S, Liebman SE, Rogers T, et al: Dietinduced obesity in $\mathrm{C} 57 \mathrm{BL} / 6 \mathrm{~J}$ mice causes increased renal lipid accumulation and glomerulosclerosis via a sterol regulatory elementbinding protein-1c-dependent pathway. J Biol Chem 2005, 280:32317-32325.
37. Wang Z, Jiang T, Li J, Proctor G, McManaman JL, Lucia S, et al: Regulation of renal lipid metabolism, lipid accumulation, and glomerulosclerosis in FVBdb/db mice with type 2 diabetes. Diabetes 2005, 54:2328-2335.

38. Bobulescu IA, Dubree M, Zhang J, McLeroy P, Moe OW: Effect of renal lipid accumulation on proximal tubule $\mathrm{Na}+/ \mathrm{H}+$ exchange and ammonium secretion. Am J Physiol Renal Physiol 2008, 294:F1315-F1322.

39. Deji N, Kume S, Araki S, Soumura M, Sugimoto T, Isshiki K, et al: Structural and functional changes in the kidneys of high-fat diet-induced obese mice. Am J Physiol Renal Physiol 2009, 296:F118-F126.

40. do Carmo JM, Tallam LS, Roberts JV, Brandon EL, Biglane J, da Silva AA, et al: Impact of obesity on renal structure and function in the presence and absence of hypertension: evidence from melanocortin-4 receptordeficient mice. Am J Physiol Regul Integr Comp Physiol 2009, 297:R803-R812.

41. Hall JE, Brands MW, Henegar JR, Shek EW: Abnormal kidney function as a cause and a consequence of obesity hypertension. Clin Exp Pharmacol Physiol 1998, 25:58-64.

42. Reisin E, Jack AV: Obesity and hypertension: mechanisms, cardio-renal consequences, and therapeutic approaches. Med Clin North Am 2009, 93:733-751.

43. Sijens PE, Edens MA, Bakker SJ, Stolk RP: MRI-determined fat content of human liver, pancreas and kidney. World J Gastroenterol 2010, 16:1993-1998.

\section{Pre-publication history}

The pre-publication history for this paper can be accessed here: http://www.biomedcentral.com/1471-2369/12/52/prepub

doi:10.1186/1471-2369-12-52

Cite this article as: Foster et al:: Development and reproducibility of a computed tomography-based measurement of renal sinus fat. BMC Nephrology 2011 12:52.

\section{Submit your next manuscript to BioMed Central and take full advantage of:}

- Convenient online submission

- Thorough peer review

- No space constraints or color figure charges

- Immediate publication on acceptance

- Inclusion in PubMed, CAS, Scopus and Google Scholar

- Research which is freely available for redistribution

Submit your manuscript at www.biomedcentral.com/submit
C) Biomed Central 